Pacific

Journal of

Mathematics

REARRANGEMENTS AND THE LOCAL INTEGRABLILITY OF MAXIMAL FUNCTIONS

Paul Alton Hagelstein 


\section{REARRANGEMENTS AND THE LOCAL INTEGRABLILITY OF MAXIMAL FUNCTIONS}

\section{Paul Alton Hagelstein}

Let $M_{\mathrm{HL}}$ and $M_{\mathrm{S}}$ respectively denote the Hardy-Littlewood and strong maximal operators, and let $M_{x}$ and $M_{y}$ respectively denote the one-dimensional Hardy-Littlewood maximal operators in the horizontal and vertical directions in $\mathbf{R}^{2}$. It is well known that if $f$ and $\widetilde{f}$ are equidistributed functions supported on $Q=[0,1] \times[0,1]$, then $\int_{Q} M_{\mathrm{HL}} f \sim \int_{Q} M_{\mathrm{HL}} \tilde{f}$. This article examines the relationships between $\int_{Q} M_{y} \tilde{f}$ and $\int_{Q} M_{y} f, \int_{Q} M_{y} M_{x} \tilde{f}$ and $\int_{Q} M_{y} M_{x} f$, and $\int_{Q} M_{\mathrm{S}} \tilde{f}$ and $\int_{Q} M_{\mathrm{S}} f$ in the scenario in which $\widetilde{f}$ and $f$ are horizontal rearrangements of one another, meaning that $\tilde{f}(\cdot, y)$ and $f(\cdot, y)$ are equidistributed on $[0,1]$ for any value of $y$.

The rearrangement results provided are not only of intrinsic interest, but also yield tools for more detailed examinations involving the local integrablility of maximal functions. They are used in a companion paper to prove that if $f$ is supported on $Q, \int_{Q} M_{y} M_{x} f<\infty$, and $\int_{Q} M_{x} M_{y} f=\infty$, then there exists a set $A$ of finite measure in $\mathrm{R}^{2}$ such that $\int_{A} M_{\mathrm{S}} f=\infty$.

We begin with the following definitions:

Definition 1 (Hardy-Littlewood maximal function). Let $f$ be a measurable function defined on $\mathbf{R}^{n}$. Let $B(p, r)$ denote the Euclidean ball in $\mathbf{R}^{n}$ centered at $p$ of radius $r$, and let $|B(p, r)|$ denote the Lebesgue measure of $B(p, r)$. The Hardy-Littlewood maximal function of $f$ is defined on $\mathbf{R}^{n}$ by

$$
M_{\mathrm{HL}} f(p)=\sup _{r>0} \frac{1}{|B(p, r)|} \int_{B(p, r)}|f(z)| d z .
$$

Definition 2 (Strong maximal function). Let $f$ be a measurable function defined on $\mathbf{R}^{2}$. The strong maximal function of $f$ is defined on $\mathbf{R}^{2}$ by

$$
M_{\mathrm{S}} f(x, y)=\sup _{\substack{x_{1}<x<x_{2} \\ y_{1}<y<y_{2}}} \frac{1}{\left(x_{2}-x_{1}\right)\left(y_{2}-y_{1}\right)} \int_{x_{1}}^{x_{2}} \int_{y_{1}}^{y_{2}}|f(u, v)| d v d u .
$$

Definition 3 (Horizontal maximal function). Let $f$ be a measurable function defined on $\mathbf{R}^{2}$. The horizontal maximal function of $f$ is defined on $\mathbf{R}^{2}$ 
by

$$
M_{x} f(u, v)=\sup _{u_{1}<u<u_{2}} \frac{1}{u_{2}-u_{1}} \int_{u_{1}}^{u_{2}}|f(w, v)| d w .
$$

Definition 4 (Vertical maximal function). Let $f$ be a measurable function defined on $\mathbf{R}^{2}$. The vertical maximal function of $f$ is defined on $\mathbf{R}^{2}$ by

$$
M_{y} f(u, v)=\sup _{v_{1}<v<v_{2}} \frac{1}{v_{2}-v_{1}} \int_{v_{1}}^{v_{2}}|f(u, w)| d w .
$$

The first rearrangement result we consider is a simple consequence of the following result due to E. M. Stein [6]:

Theorem 5. Let $f$ be a measurable function supported on $I^{n}$, the unit $n$ cube in $\mathbf{R}^{n}$. There exist positive, finite constants $c, C$ (depending on $n$ ) such that

$$
c\|f\|_{L \log L\left(I^{n}\right)} \leq \int_{I^{(n)}} M_{\mathrm{HL}} f \leq C\|f\|_{L \log L\left(I^{n}\right)} .
$$

Inequalities such as (5) will often be denoted by

$$
\|f\|_{L \log L\left(I^{n}\right)} \sim \int_{I^{n}} M_{\mathrm{HL}} f
$$

for the remainder of this paper. Also, the unit square $I^{2}$ in $\mathbf{R}^{2}$ will be denoted by $Q$.

Corollary 6. Let $f$ and $\widetilde{f}$ be equidistributed functions supported on $I^{n}$. Then

$$
\int_{I^{n}} M_{\mathrm{HL}} f \sim \int_{I^{n}} M_{\mathrm{HL}} \tilde{f}
$$

Proof. As $\|f\|_{L \log L\left(I^{n}\right)}=\|\widetilde{f}\|_{L \log L\left(I^{n}\right)}$, this follows directly from Theorem 5 .

Corollary 7. Suppose $f$ and $\widetilde{f}$ are functions supported on $Q$ and also suppose that $f(\cdot, y)$ and $\widetilde{f}(\cdot, y)$ are equidistributed for each $y \in[0,1]$. Then

$$
\int_{Q} M_{x} \widetilde{f} \sim \int_{Q} M_{x} f
$$

Proof. This is an application of Corollary 6 and the Fubini theorem.

Far more interesting is the relationship between $\int_{Q} M_{y} f$ and $\int_{Q} M_{y} \tilde{f}$ when $f$ and $\widetilde{f}$ are horizontal rearrangements of each other, i.e., $\tilde{f}(\cdot, y)$ and $f(\cdot, y)$ are equidistributed on $[0,1]$ for any value of $y$. In general these integrals are not comparable. For example, define $g$ by

$$
g(x, y)=\frac{1}{|x-y|(\log |x-y|)^{2}} \chi_{Q} .
$$


Let $\widetilde{g}$ be the function supported on $Q$ which is a horizontal rearrangement of $g$ and such that $\widetilde{g}(x, y)$ is nonincreasing in $x$, i.e., $\widetilde{g}\left(x_{1}, y\right) \geq \widetilde{g}\left(x_{2}, y\right)$ for any $0 \leq x_{1} \leq x_{2} \leq 1$ and any value of $y$. One can readily compute that $\int_{Q} M_{y} \widetilde{g}<\infty$ but $\int_{Q} M_{y} g=\infty$. We do have the following result, however:

Theorem 8. Let $f$ be a nonnegative measurable function supported on $Q$. Let $\widetilde{f}$ be the function supported on $Q$ which is nonincreasing in $x$ and such that, for each $y \in[0,1], \widetilde{f}(\cdot, y)$ and $f(\cdot, y)$ are equidistributed.

Then

$$
\int_{Q} M_{y} \widetilde{f} \leq c \int_{Q} M_{y} f
$$

where $c$ is a universal constant.

Proof. Let $\alpha>0$. Let $\lambda(\alpha)=\left|\left\{(u, v) \in Q: M_{y} f(u, v)>\alpha\right\}\right|$. Define $\widetilde{\lambda}(\alpha)$ similarly. It suffices to show that $\tilde{\lambda}(\alpha) \leq 400 \lambda(\alpha / 64)$.

Without loss of generality, assume $f$ is smooth on $Q$. Take the CalderónZygmund decomposition of $f$ with respect to $\alpha$ on each vertical segment in $\{s \times[0,1], s \in[0,1]\}$ of $Q$, yielding for each $x \in[0,1]$ disjoint sets $Q_{x, j, \alpha} \subseteq[0,1]$ such that $\alpha<\left|Q_{x, j, \alpha}\right|^{-1} \int_{Q_{x, j, \alpha}} f(x, z) d z \leq 2 \alpha$. (In the case that $\int_{0}^{1} f(x, z) d z>2 \alpha$, set $Q_{x, 1, \alpha}=[0,1]$.) Note that $f(p) \leq \alpha$ for almost every $p$ in the complement of $\bigcup_{x \in[0,1], j \in \mathbf{Z}_{+}}\left(x \times Q_{x, j, \alpha}\right)$. For $\tilde{f}$ one may produce the associated sets $\widetilde{Q}_{x, j, \alpha}$ in a similar fashion.

Let $E_{\alpha}=\left\{(x, y) \in Q: y \in \bigcup_{x \in[0,1], j \in \mathbf{Z}_{+}} Q_{x, j, \alpha}\right\}$. Define $\widetilde{E}_{\alpha}$ similarly. It suffices to show that $\left|\widetilde{E}_{4 \alpha}\right| \leq 2\left|E_{\alpha}\right|$. To see this, recall from the theory of the Hardy-Littlewood maximal operator $M_{\mathrm{HL}}$ that if $g$ is a measurable function supported on the unit interval $[0,1], g \geq 0, \int_{0}^{1} g \leq 2 \alpha$,

$$
\lambda_{\mathrm{HL}}(\alpha)=\left|\left\{x \in[0,1]: M_{\mathrm{HL}} g(x)>\alpha\right\}\right|,
$$

and $E_{\mathrm{HL}, \alpha}=\bigcup Q_{j, \alpha}$, where the $Q_{j, \alpha}$ are the intervals obtained by taking the Calderón-Zygmund decomposition of $g$ with respect to $\alpha$, then $\left|E_{\mathrm{HL}, \alpha}\right| \leq$ $\lambda_{\mathrm{HL}}(\alpha / 2) \leq 200\left|E_{\mathrm{HL}, \alpha / 8}\right|$. This readily yields that, if $\left|\widetilde{E}_{4 \alpha}\right| \leq 2\left|E_{\alpha}\right|$, then $\widetilde{\lambda}(\alpha) \leq 200\left|\widetilde{E}_{\alpha / 8}\right| \leq 400\left|E_{\alpha / 32}\right| \leq 400 \lambda(\alpha / 64)$. Hence $\widetilde{\lambda}(\alpha) \leq 400 \lambda(\alpha / 64)$, as desired.

To show that $\left|\widetilde{E}_{4 \alpha}\right| \leq 2\left|E_{\alpha}\right|$ we proceed as follows:

First we consider the special case in which $\int_{0}^{1} f(x, y) d y \leq \alpha$ for any $x \in[0,1]$. Having taken the Calderón-Zygmund decomposition of $f$ with respect to $\alpha$ described above, we obtain the disjoint sets $Q_{x, j, \alpha} \subset[0,1]$ for each $x \in[0,1]$ and the associated set $E_{\alpha}$. Now, $f(p) \leq \alpha$ for almost every $p$ in the complement of $E_{\alpha}$. So if $S$ is a measurable subset of $Q$ and $|S|>2\left|E_{\alpha}\right|$, then $\int_{S} f \leq 2 \alpha|S|$. Now let $\phi: Q \rightarrow Q$ be a measure-preserving bijection such that $\widetilde{f}(\phi(p))=f(p)$ for any $p \in Q$. Using $\phi$ we see that $\left|\widetilde{E}_{4 \alpha}\right| \leq 2\left|E_{\alpha}\right|$. Otherwise, if $\left|\widetilde{E}_{4 \alpha}\right|>2\left|E_{\alpha}\right|$ we would have $\left|\widetilde{E}_{4 \alpha}\right|^{-1} \int_{\widetilde{E}_{4 \alpha}} \widetilde{f}=$ 
$\left|\widetilde{E}_{4 \alpha}\right|^{-1} \int_{\phi^{-1}\left(\widetilde{E}_{4 \alpha}\right)} f \leq 2 \alpha$ by the above. But $\left|\widetilde{E}_{4 \alpha}\right|^{-1} \int_{\widetilde{E}_{4 \alpha}} \widetilde{f}>4 \alpha$ by the construction of $\widetilde{E}_{4 \alpha}$. So $\left|\widetilde{E}_{4 \alpha}\right| \leq 2\left|E_{\alpha}\right|$ if $\int_{0}^{1} f(x, y) d y \leq \alpha$ for all $x \in[0,1]$.

Now we let $f$ be an arbitrary nonnegative smooth function on $Q$. Without loss of generality assume there exists $c \in(0,1)$ such that $\int_{0}^{1} f(x, y) d y>\alpha$ if $x<c$, and $\int_{0}^{1} f(x, y) d y \leq \alpha$ if $x \geq c$. Form the Calderón-Zygmund decomposition of $f$ with respect to $\alpha$ as before, obtaining the $Q_{x, j, \alpha}$ and $E_{\alpha}$. Note that $Q_{x, 1, \alpha}=[0,1]$ if $x<c$.

For each $y \in[0,1]$ we define the functions $f_{y}(x)$ on $[0,1]$ by $f_{y}(x)=$ $f(x, y)$. We construct a function $f_{y}^{\prime}(x)$ on $[0,1]$ equidistributed to $f_{y}(x)$ such that $f_{y}^{\prime}\left(x_{2}\right) \leq f_{y}^{\prime}\left(x_{1}\right)$ if $x_{2} \geq c \geq x_{1}$ and $f_{y}^{\prime}(x) \leq f_{y}(x)$ if $x \geq c$ as follows:

Let $B_{y}=\left\{x \in[0, c): f_{y}(x)<\widetilde{f}_{y}(c)\right\}$.

Let $A_{y} \subset\left\{x \in[c, 1]: f_{y}(x) \geq \widetilde{f}_{y}(c)\right\}$ be such that the measure of its interior is equal to $\left|B_{y}\right|$. Let $A_{y}^{\circ}$ and $B_{y}^{\circ}$ respectively denote the interiors of $A_{y}$ and $B_{y}$. Let $\phi_{y}: A_{y}^{\circ} \rightarrow B_{y}^{\circ}$ be a measure-preserving bijection such that if $x \in A_{y}^{\circ},\left|\left\{b \in B_{y}^{\circ}: b<\phi_{y}(x)\right\}\right|=\left|\left\{a \in A_{y}^{\circ}: a<x\right\}\right|$. Define $f_{y}^{\prime}(x)$ by

$$
f_{y}^{\prime}(x)= \begin{cases}f_{y}(x) & \text { if } x \notin A_{y}^{\circ} \cup B_{y}^{\circ}, \\ f_{y}\left(\phi_{y}^{-1}(x)\right) & \text { if } x \in B_{y}^{\circ}, \\ f_{y}\left(\phi_{y}(x)\right) & \text { if } x \in A_{y}^{\circ} .\end{cases}
$$

Note that $f_{y}^{\prime}(x) \leq f_{y}(x)$ if $x>c$. Define the function $f^{\prime}$ on $Q$ by $f^{\prime}(x, y)=$ $f_{y}^{\prime}(x)$. For the Calderón-Zygmund decomposition of $f^{\prime}$ with respect to $\alpha$ as above, obtaining the associated sets $Q_{x, j, \alpha}^{\prime}, E_{\alpha}^{\prime}$. Note that $E_{\alpha} \supseteq E_{\alpha}^{\prime}$, so without loss of generality we may assume $f=f^{\prime}$. Hence, without loss of generality, $f\left(x_{1}, y\right) \geq f\left(x_{2}, y\right)$ if $0 \leq x_{1}<c \leq x_{2} \leq 1$, and $\int_{0}^{1} f(x, y) d y>\alpha$ if and only if $x<c$.

Let $f_{1}=f \chi_{[0 \leq x<c]}, f_{2}=f \chi_{[c \leq x \leq 1]}$. So $f=f_{1}+f_{2}$. Let $\tilde{f}_{1}$ be a rearrangement of $f_{1}$ such that, for each $y \in[0,1], f_{1}(\cdot, y)$ and $\widetilde{f}_{1}(\cdot, y)$ are equidistributed and $\widetilde{f}_{1}(x, y)$ is nonincreasing in $x$. Define $\widetilde{f}_{2}$ to be the rearrangement of $f_{2}$ within $\{Q \cap\{(x, y): c \leq x \leq 1\}\}$ such that, for each $y \in[\underset{\sim}{0}, 1]$, the functions $f_{2}(\cdot, y)$ and $\widetilde{f}_{2}(\cdot, y)$ are equidistributed and such that $\widetilde{f}_{2}(x, y)$ is nonincreasing in $x$ in $\{x: c \leq x \leq 1\}$. Now $\widetilde{f}=\widetilde{f}_{1}+\widetilde{f}_{2}$. Let

$$
E_{1, \alpha}=\bigcup_{\substack{x \in[0, c) \\ j \in \mathbf{Z}_{+}}}\left(x \times Q_{x, j, \alpha}\right), \quad E_{2, \alpha}=\bigcup_{\substack{x \in[c, 1] \\ j \in \mathbf{Z}_{+}}}\left(x \times Q_{x, j, \alpha}\right) .
$$

Define $\widetilde{E}_{1, \alpha}$ and $\widetilde{E}_{2, \alpha}$ similarly. Note that $\left|\widetilde{E}_{1,4 \alpha}\right| \leq 2\left|E_{1, \alpha}\right|$ trivially (as $Q_{x, 1, \alpha}=[0,1]$ if $\left.x<c\right)$ and $\left|\widetilde{E}_{2,4 \alpha}\right| \leq 2\left|E_{2, \alpha}\right|$ by the special case argument, since $\int_{0}^{1} f(x, y) d y \leq \alpha$ for $x \geq c$. As $\left|E_{\alpha}\right|=\left|E_{1, \alpha}\right|+\left|E_{2, \alpha}\right|$ and $\left|\widetilde{E}_{\alpha}\right|=$ $\left|\widetilde{E}_{1, \alpha}\right|+\left|\widetilde{E}_{2, \alpha}\right|$, we see that $\left|\widetilde{E}_{4 \alpha}\right| \leq 2\left|E_{\alpha}\right|$, as desired. 
Theorem 8 yields the following two easy but useful corollaries:

Corollary 9. Let $f$ be a nonnegative measurable function supported on $Q$. Let $\widetilde{f}(x, y)$ be the function supported on $Q$ which is nonincreasing in $x$ and such that, for each $y \in[0,1], \widetilde{f}(\cdot, y)$ and $f(\cdot, y)$ are equidistributed. Then

$$
\int_{Q} M_{\mathrm{HL}} M_{y} \tilde{f} \leq c \int_{Q} M_{\mathrm{HL}} M_{y} f
$$

where $c$ is a universal constant.

Proof. Define $\lambda(\alpha), \widetilde{\lambda}(\alpha)$ as in the proof of Theorem 8. As the proof of Theorem 8 indicated that $\tilde{\lambda}(\alpha) \lesssim 400 \lambda(\alpha / 64)$, we see that $\left\|M_{y} \widetilde{f}\right\|_{L \log L} \lesssim$ $\left\|M_{y} f\right\|_{L \log L}$. Hence Theorem 5 yields $\int_{Q} M_{\mathrm{HL}} M_{y} \widetilde{f} \lesssim \int_{Q} M_{\mathrm{HL}} M_{y} f$.

Corollary 10. Let $f$ be a nonnegative measurable function supported on $Q$. Let $\widetilde{f}$ be a function on $Q$ which is nonincreasing in $x$ and such that, for each $y \in[0,1], \widetilde{f}(\cdot, y)$ and $f(\cdot, y)$ are equidistributed. Then

$$
\int_{Q} M_{y} M_{x} \widetilde{f} \leq c \int_{Q} M_{y} M_{x} f
$$

where $c$ is a universal constant.

Proof. By Theorem $8, \int_{Q} M_{y}\left(\widetilde{M_{x} f}\right) \leq c \int_{Q} M_{y} M_{x} f$. So it suffices to show that $\int_{Q} M_{y} M_{x} \widetilde{f} \leq c \int_{Q} M_{y}\left(\widetilde{M_{x} f}\right)$. To do this, it suffices to show that, given a nonnegative function $g$ supported on $[0,1]$ and $p \in(0,1)$, we have $\widetilde{M_{\mathrm{HL}} g}(p) \geq c M_{\mathrm{HL}} \widetilde{g}(p)$, where $c$ is independent of $g$ and $p$, and $\widetilde{g}$ is the function supported on $[0,1]$ which is equidistributed to $g$ and such that $\widetilde{g}\left(x_{1}\right) \geq \widetilde{g}\left(x_{2}\right)$ whenever $0<x_{1} \leq x_{2}<1$. Well, suppose $M_{\mathrm{HL}} \widetilde{g}(p)=\alpha$. Then on a set $A \subseteq[0,1]$ of measure $|A|=|p|$, we have $|A|^{-1} \int_{A}|g|=\alpha$. Assuming without loss of generality that $\int_{0}^{1} g \leq \alpha / 2$, we take a CalderónZygmund decomposition of $g$ with respect to $\alpha / 4$, yielding intervals $Q_{j, \alpha / 4}$.

Now $\left|\bigcup Q_{j, \alpha / 4}\right| \geq|A|$. Otherwise we would have $\left|\bigcup Q_{j, \alpha / 4}\right|<|A|$, implying that if $E$ is a set contained in $[0,1]$ such that $|E| \geq|A|$,

$$
\begin{aligned}
\frac{1}{|E|} \int_{E}|g| & \leq \frac{1}{|E|}\left(|E| \frac{\alpha}{2}+\left|\bigcup Q_{j, \frac{\alpha}{4}}\right| \frac{\alpha}{2}\right)=\frac{\alpha}{2}\left(1+\frac{\left|\bigcup Q_{j, \frac{\alpha}{4}}\right|}{|E|}\right) \\
& <\frac{\alpha}{2}(1+1)=\alpha,
\end{aligned}
$$

contradicting the fact that $|A|^{-1} \int_{A}|g|=\alpha$.

So $M_{\mathrm{HL}} g>\alpha / 8$ on a set contained in $[0,1]$ of measure greater than or equal to $|A|$. So $\widetilde{M_{\mathrm{HL}} g}(p)>\alpha / 8$. Hence $\widetilde{M_{\mathrm{HL}} g}(p) \geq \frac{1}{8} M_{\mathrm{HL}} \widetilde{g}(p)$.

The statement of Corollary 10 is false if the operator $M_{y} M_{x}$ is replaced by either $M_{x} M_{y}$ or $M_{\mathrm{S}}$. To exhibit a counterexample, we first define the 
functions $h_{2^{n}}$ as follows:

$$
h_{2^{n}}(x, y)=\sum_{m=0}^{2^{n}-1} 2^{2^{n}-m-1} \chi_{\left[0,2^{-2^{n}+m+1}\right]}(y) \chi_{\left[m \cdot 2^{-n},(m+1) \cdot 2^{-n}\right]}(x) .
$$

Let now the function $h$ be defined by

$$
h=\sum_{k=1}^{\infty} \frac{1}{2^{k+1}} \frac{1}{2^{4^{k-1}}} h_{2^{4^{k-1}}} .
$$

One can show that, if $\widetilde{h}(x, y)$ is a horizontal rearrangement of $h$ which is nonincreasing in $x$, then $\int_{Q} M_{x} M_{y} h=\int_{Q} M_{\mathrm{S}} h<\infty$, but $\int_{Q} M_{x} M_{y} \widetilde{h}$ and $\int_{Q} M_{\mathrm{S}} \widetilde{h}$ are infinite. More details in this regard are found in [4].

We now consider rearrangement results involving sums of maximal operators. A good example of such a result is the following:

Theorem 11. Let $f$ and $\widetilde{f}$ be equidistributed functions supported on $Q$. Then

$$
\int_{Q}\left(M_{x} f+M_{y} f\right) \sim \int_{Q}\left(M_{x} \widetilde{f}+M_{y} \widetilde{f}\right) .
$$

Proof. In [3] it is shown that if $f$ is supported on $Q$, then

$$
\int_{Q} M_{\mathrm{HL}} f \sim \int_{Q} M_{x} f+M_{y} f .
$$

The result then follows from Theorem 5 .

We now strengthen this result. Define the maximal operators $M, \bar{M}$ as follows:

Definition 12. Let $f$ be a measurable function supported on $Q$. The associated maximal function $M f$ is defined on $Q$ by

$$
M f\left(p_{1}, p_{2}\right)=\sup _{x_{1}<p_{1}<x_{2}} \frac{1}{x_{2}-x_{1}} \int_{x_{1}}^{x_{2}} \int_{0}^{1}|f(x, y)| d y d x .
$$

The associated maximal function $\bar{M} f$ is defined on $Q$ by

$$
\bar{M} f\left(p_{1}, p_{2}\right)=\sup _{y_{1}<p_{2}<y_{2}} \frac{1}{y_{2}-y_{1}} \int_{0}^{1} \int_{y_{1}}^{y_{2}}|f(x, y)| d y d x .
$$

Theorem 13. Let $f$ and $f^{*}$ be equidistributed functions supported on $Q$. Then

$$
\int_{Q}\left(M f+M_{y} f\right) \sim \int_{Q}\left(M f^{*}+M_{y} f^{*}\right) .
$$


Moreover,

$$
\int_{Q} M_{\mathrm{HL}} f \sim \int_{Q}\left(M f+M_{y} f\right) .
$$

Proof. By Theorem 5, we need only to show (12). Now, by a theorem of Jessen, Marcinkiewicz, and Zygmund [5] we already know that

$$
\int_{Q}\left(M f+M_{y} f\right) \leq \int_{Q}\left(M_{x} f+M_{y} f\right) \lesssim\|f\|_{L \log L(Q)} \sim \int_{Q} M_{\mathrm{HL}} f .
$$

So it suffices to show that $\int_{Q} M_{\mathrm{HL}} f \lesssim \int_{Q} M f+M_{y} f$.

We may assume without loss of generality that $f$ is smooth and nonnegative. Let $\widetilde{f}(x, y)$ be the function supported on $Q$ which is nonincreasing in $x$ and such that, for each $y \in[0,1], \widetilde{f}(\cdot, y)$ and $f(\cdot, y)$ are equidistributed. It is enough to show that $\int_{Q} M \tilde{f} \lesssim \int_{Q}\left(M f+M_{y} f\right)$. For then

$$
\begin{aligned}
\|f\|_{L \log L(Q)} & =\|\widetilde{f}\|_{L \log L(Q)} \\
& \sim \int_{Q} M_{\mathrm{HL}} \tilde{f} \quad(\text { by Theorem } 5) \\
& \sim \int_{Q}\left(M_{x} \tilde{f}+M_{y} \widetilde{f}\right) \quad(\text { by }(10)) \\
& =\int_{Q} M \widetilde{f}+\int_{Q} M_{y} \widetilde{f} \\
& \lesssim \int_{Q}\left(M f+M_{y} f\right)+\int_{Q} M_{y} \widetilde{f} \\
& \lesssim \int_{Q}\left(M f+M_{y} f\right) \quad \text { (by Theorem 8). }
\end{aligned}
$$
that

We now show $\int_{Q} M \widetilde{f} \lesssim \int_{Q}\left(M f+M_{y} f\right)$. To do this, it suffices to show

$$
|\{p \in Q: M \tilde{f}(p)>2 \alpha\}| \leq 2\left|\left\{p \in Q: M f(p)+M_{y} f(p)>\alpha\right\}\right|
$$

for each $\alpha>0$.

Now, let $A=\{p \in Q: M f(p)>\alpha\}$.

Let $B=\left\{p \in Q: p \notin A, M_{y} f(p)>\alpha\right\}$. If $A=Q$, we're done. Otherwise, $\frac{1}{|A|} \int_{A}|f| \leq 2 \alpha$. Also, if $p \notin A \cup B$, then $f(p) \leq \alpha$. Note also that $\frac{1}{|B|} \int_{B} f \leq$ $2 \alpha$.

Now, let $f^{\prime}$ be a function supported on $Q$ which is equidistributed to $f$ and such that $f^{\prime}\left(x_{1}, y_{1}\right) \geq f^{\prime}\left(x_{2}, y_{2}\right)$ whenever $0 \leq x_{1} \leq x_{2} \leq 1,0 \leq y_{1} \leq 1$, and $0 \leq y_{2} \leq 1$. Note if $p \in Q, M f^{\prime}(p) \geq M \widetilde{f}(p)$. So it suffices to show

$$
\left|\left\{p \in Q: M f^{\prime}(p)>2 \alpha\right\}\right| \leq 2\left|\left\{p \in Q: M f(p)+M_{y} f(p)>\alpha\right\}\right| .
$$


Let $D=[0,|A \cup B|] \times[0,1]$ be a subset of $Q$. Note that $|D|^{-1} \int_{D} f^{\prime} \leq 2 \alpha$. If $p \notin D$, then $f^{\prime}(p) \leq \alpha$. So if $p \notin D, M f^{\prime}(p) \leq 2 \alpha$. So

$$
\begin{aligned}
\left|\left\{p \in Q: M f^{\prime}(p)>2 \alpha\right\}\right| & \leq|A|+|B| \\
& \leq|\{p \in Q: M f(p)>\alpha\}|+\left|\left\{p \in Q: M_{y} f(p)>\alpha\right\}\right| \\
& \leq 2\left|\left\{p \in Q: M f(p)+M_{y} f(p)>\alpha\right\}\right| .
\end{aligned}
$$

We now turn to a substantially more sophisticated rearrangement result involving sums of iterated maximal operators. The following result, besides being of intrinsic interest, is used in [4] to show that for any measurable function $f$ supported on $Q$,

$$
\|f\|_{L(\log L)^{2}(Q)} \sim \int_{Q}\left(M M f+\bar{M} \bar{M} f+M_{y} M_{x} f\right) .
$$

This is in turn used to show that if $f$ is a function supported on $Q$ such that $\int_{Q} M_{y} M_{x} f<\infty$ but $\int_{Q} M_{x} M_{y} f=\infty$ (such functions do exist: the function $f(x, y)=h(y, x)$, where $h$ is defined as in (7), (8) provides an example), then there exists a set $A$ of finite measure in $\mathbf{R}^{2}$ such that $\int_{A} M_{\mathrm{S}} f=\infty$. This result is particularly striking considering that there exists a function $g$ constructed by M. E. Gomez [2] such that $g$ is supported in $Q, \int_{Q} M_{x} M_{y} g=$ $\infty$, and $\int_{Q} M_{y} M_{x} g=\infty$, but $M_{\mathrm{S}} g$ is integrable over every set of finite measure in $\mathbf{R}^{2}$.

Theorem 14. Let $f$ be a measurable function supported on $Q$. Let $f^{\prime}$ be a function supported on $Q$ such that $f^{\prime}(\cdot, y)$ and $f(\cdot, y)$ are equidistributed for each $y \in[0,1]$. Then

$$
\int_{Q}\left(M M f^{\prime}+M_{y} M_{x} f^{\prime}\right) \sim \int_{Q}\left(M M f+M_{y} M_{x} f\right) .
$$

Proof. Without loss of generality, assume $f$ is nonnegative. Let $\tilde{f}$ be a function supported on $Q$ which is nonincreasing in $x$ and such that $\widetilde{f}(\cdot, y)$ and $f(\cdot, y)$ are equidistributed for each $y \in[0,1]$. It suffices to show that

$$
\int_{Q}\left(M M \widetilde{f}+M_{y} M_{x} \widetilde{f}\right) \sim \int_{Q}\left(M M f+M_{y} M_{x} f\right) .
$$

Well,

$$
\begin{aligned}
\int_{Q}\left(M M f+M_{y} M_{x} f\right) \lesssim \int_{Q}\left(M_{x} M_{x} f+M_{y} M_{x} f\right) & \sim \int_{Q} M_{\mathrm{HL}} M_{x} f \\
& \sim \int_{Q} M_{\mathrm{HL}} M_{x} \widetilde{f}
\end{aligned}
$$


(the last step by Theorem 5, since $\left\|M_{x} f\right\|_{L \log L} \sim\left\|M_{x} \widetilde{f}\right\|_{L \log L}$ ). Further,

$$
\begin{aligned}
\int_{Q} M_{\mathrm{HL}} M_{x} \tilde{f} & \sim \int_{Q}\left(M_{x} M_{x} \tilde{f}+M_{y} M_{x} \widetilde{f}\right) \quad(\text { by }(10)) \\
& =\int_{Q}\left(M M \widetilde{f}+M_{y} M_{x} \widetilde{f}\right) .
\end{aligned}
$$

So

$$
\int_{Q}\left(M M f+M_{y} M_{x} f\right) \lesssim \int_{Q}\left(M M \widetilde{f}+M_{y} M_{x} \widetilde{f}\right) .
$$

It suffices then to prove the reverse of this last inequality. This step is somewhat involved and will be the focus of the remainder of this paper.

It will be technically convenient to work with the dyadic analogues of the maximal operators $M_{\mathrm{HL}}, M, \bar{M}, M_{x}$, and $M_{y}$. Recall that a dyadic interval in $[0,1]$ is an interval of the form $\left[k \cdot 2^{j},(k+1) \cdot 2^{j}\right]$, where $j$ is a nonpositive integer and $k$ is a nonnegative integer such that $(k+1) \cdot 2^{j} \leq 1$. We denote the set of dyadic subintervals of $[0,1]$ by $\mathcal{I}^{\Delta}$. A dyadic square in $Q$ is a set of the form $I \times J$, where $I$ and $J$ are dyadic intervals in $[0,1]$ of the same length. We denote the set of dyadic squares in $Q$ by $\mathcal{S}^{\Delta}$. We formally define the dyadic maximal operators $M_{\mathrm{HL}}{ }^{\Delta}, M^{\Delta}, \bar{M}^{\Delta}, M_{x}^{\Delta}$, and $M_{y}^{\Delta}$ as follows:

Definition 15. Let $f$ be a measurable function supported on $Q$. The dyadic Hardy-Littlewood maximal function $M_{\mathrm{HL}}^{\Delta} f$ is defined on $Q$ by

$$
M_{\mathrm{HL}}^{\Delta} f(p)=\sup _{p \in S \in \mathcal{S}^{\Delta}} \frac{1}{|S|} \int_{S}|f| .
$$

The maximal function $M^{\Delta} f$ is defined by

$$
M^{\Delta} f\left(p_{1}, p_{2}\right)=\sup _{p_{1} \in I \in \mathcal{I} \Delta} \frac{1}{|I|} \int_{I} \int_{0}^{1}|f(x, y)| d y d x .
$$

Similarly, we define $\bar{M}^{\Delta} f, M_{x}^{\Delta} f$ and $M_{y}^{\Delta} f$ by

$$
\begin{aligned}
& \bar{M}^{\Delta} f\left(p_{1}, p_{2}\right)=\sup _{p_{2} \in I \in \mathcal{I}^{\Delta}} \frac{1}{|I|} \int_{0}^{1} \int_{I}|f(x, y)| d y d x, \\
& M_{x}^{\Delta} f\left(p_{1}, p_{2}\right)=\sup _{p_{1} \in I \in \mathcal{I}^{\Delta}} \frac{1}{|I|} \int_{I}\left|f\left(x, p_{2}\right)\right| d x, \\
& M_{y}^{\Delta} f\left(p_{1}, p_{2}\right)=\sup _{p_{2} \in I \in \mathcal{I}^{\Delta}} \frac{1}{|I|} \int_{I}\left|f\left(p_{1}, y\right)\right| d y .
\end{aligned}
$$

The dyadic Hardy-Littlewood maximal function $M_{\mathrm{HL}}^{\Delta} f$ of a measurable function $f$ supported on $[0,1]$ is defined on $[0,1]$ by

$$
M_{\mathrm{HL}}^{\Delta} f(p)=\sup _{p \in I \in \mathcal{I}^{\Delta}} \frac{1}{|I|} \int_{I}|f| .
$$


We will also require the following definition and theorem introduced in $[\mathbf{3}]$ : Definition 16. Let $\beta$ be a countable collection of Lebesgue measurable subsets of the unit $n$-cube $I^{n}$ in $\mathbf{R}^{n}$ of positive measure. A (possibly finite) subset $\left\{R_{i}\right\}$ of $\beta$ is said to be a Córdoba-Fefferman collection with respect to $\beta$ if there exists an enumeration $\widetilde{R}_{1}, \widetilde{R}_{2}, \widetilde{R}_{3}, \ldots$ of the elements of $\left\{R_{i}\right\}$ such that $\left|\widetilde{R}_{i} \cap \bigcup_{j<i} \widetilde{R}_{j}\right| \leq \frac{1}{2}\left|\widetilde{R}_{i}\right|$ for each $i$. In this case we write $\left\{R_{i}\right\} \in \operatorname{CFC}(\beta)$.

Theorem 17. Let $\beta$ be a countable collection of Lebesgue measurable subsets of the unit $n$-cube $I^{n}$ in $\mathbf{R}^{n}$ of positive measure. Let $\beta$ be such that for any point $x$ in $I^{n}, x \in R$ for some $R \in \beta$. Define the maximal operator $M_{\beta}$ on $L^{1}\left(I^{n}\right)$ by

$$
M_{\beta} f(x)=\sup _{x \in R \in \beta} \frac{1}{|R|} \int_{R}|f(y)| d y .
$$

Suppose $M_{\beta}$ satisfies the (Tauberian) condition

$$
\left|\left\{x \in I^{n}: M_{\beta} \chi_{E}(x) \geq \frac{1}{2}\right\}\right| \leq C_{\beta}|E|
$$

for all measurable sets $E \subset I^{n}$. Then if $f \in L^{1}\left(I^{n}\right)$,

$$
\int_{I^{n}} M_{\beta} f \sim \sup _{\left\{R_{i}\right\} \in \operatorname{CFC}(\beta)} \int_{I^{n}}|f| \sum_{i} \chi_{R_{i}}
$$

In particular,

$$
\frac{1}{2} \sup _{\left\{R_{i}\right\} \in \mathrm{CFC}(\beta)} \int_{I^{n}}|f| \sum_{i} \chi_{R_{i}} \leq \int_{I^{n}} M_{\beta} f \leq 4 C_{\beta} \sup _{\left\{R_{i}\right\} \in \mathrm{CFC}(\beta)} \int_{I^{n}}|f| \sum_{i} \chi_{R_{i}} .
$$

The maximal operators $M, \bar{M}, M_{\mathrm{HL}}, M_{\mathrm{S}}$, and their corresponding dyadic analogues satisfy the desired Tauberian condition, since $M_{\mathrm{HL}}$ is of weak type $(1,1)$. Also, as a matter of notation, if a given maximal operator $M_{\beta}$ is naturally associated to a collection $\beta$, as, say, $M_{\mathrm{S}}$ is associated to the set of rectangles with sides parallel to the axes, we will frequently denote the Córdoba-Fefferman collection $\mathrm{CFC}(\beta)$ by $\mathrm{CFC}\left(M_{\beta}\right)$.

We will need the the following lemma:

Lemma 18. Suppose $f$ is a measurable function supported on $Q$. Then

$$
\int_{Q} M^{\Delta} M_{x}^{\Delta} f \lesssim \int_{Q} M^{\Delta} M^{\Delta} f+\int_{Q} M_{y}^{\Delta} M_{x}^{\Delta} f .
$$

Proof. By Equations (10) and (12) we realize

$$
\int_{Q} M_{x} f \lesssim \int_{Q} M f+\int_{Q} M_{y} f
$$

Since $\int_{Q} M_{x} f \sim \int_{Q} M_{x}^{\Delta} f$, and in view of the inequality

$$
\left|\left\{p \in Q: M_{x} f(p)>\alpha\right\}\right|<100\left|\left\{p \in Q: M_{x}^{\Delta} f(p)>\frac{1}{100} \alpha\right\}\right|
$$


valid for all $\alpha>0$, we see by symmetry and the Fubini theorem that

$$
\int_{Q} M_{x}^{\Delta} f \lesssim \int_{Q} M^{\Delta} f+\int_{Q} M_{y}^{\Delta} f
$$

Hence

$$
\int_{Q} M_{x}^{\Delta} M_{x}^{\Delta} f \lesssim \int_{Q} M^{\Delta} M_{x}^{\Delta} f+\int_{Q} M_{y}^{\Delta} M_{x}^{\Delta} f
$$

If $\int_{Q} M_{x}^{\Delta} M_{x}^{\Delta} f \lesssim \int_{Q} M_{y}^{\Delta} M_{x}^{\Delta} f$ we're done, since $\int_{Q} M^{\Delta} M_{x}^{\Delta} f \lesssim \int_{Q} M_{x}^{\Delta} M_{x}^{\Delta} f$. So we may assume without loss of generality that

$$
\int_{Q} M_{x}^{\Delta} M_{x}^{\Delta} f \lesssim \int_{Q} M^{\Delta} M_{x}^{\Delta} f
$$

Hence it is enough to prove the following:

Lemma 19. Suppose $f$ is a measurable function supported on $Q$ such that $\int_{Q} M_{x}^{\Delta} M_{x}^{\Delta} f \lesssim \int_{Q} M^{\Delta} M_{x}^{\Delta} f$. Then $\int_{Q} M_{x}^{\Delta} M_{x}^{\Delta} f \lesssim \int_{Q} M^{\Delta} M^{\Delta} f$.

Proof. We first prove the following claim:

Claim 20. Suppose $f \in L^{\infty}([0,1])$. Let $\epsilon>0$. Then there exists $\left\{R_{i}\right\} \in$ $\mathrm{CFC}\left(M_{\mathrm{HL}}^{\Delta}\right)$ such that:

(i) $M_{\mathrm{HL}}^{\Delta} f \sim \sum_{i} \frac{1}{\left|R_{i}\right|}\left(\int_{R_{i}}|f|\right) \chi_{R_{i}}$ in $[0,1]$ except on a set of measure less than $\epsilon$; and

(ii) $\int_{0}^{1} M_{\mathrm{HL}}^{\Delta} M_{\mathrm{HL}}^{\Delta} f \sim \int_{0}^{1}|f|\left(\sum_{i} \chi_{R_{i}}\right)^{2}$.

Proof. We may assume without loss of generality that $f$ is a smooth nonnegative function and $\|f\|_{L(\log L)^{2}([0,1])}=1$. Hence $\|f\|_{1} \leq 1$. Let $\ell$ be the largest integer such that $2^{\ell}<\|f\|_{1}$. Take the Calderón-Zygmund decomposition of $f$ with respect to $2^{\ell}$, yielding intervals $\left\{Q_{1, i}^{\prime}\right\}_{i \in \mathbf{Z}_{+}}$. Let $\left\{Q_{1, i}\right\}_{i=1, \ldots, j_{1}} \subset\left\{Q_{1, i}^{\prime}\right\}_{i \in \mathbf{Z}_{+}}$such that

$$
\left|\bigcup_{i=1}^{\infty} Q_{1, i}^{\prime} \backslash \bigcup_{i=1}^{j_{1}} Q_{1, i}\right|<\epsilon / 2 .
$$

(Note here that $Q_{1,1}=[0,1]$, and $j_{1}=1$.)

We continue by induction. Suppose $\left\{Q_{k, i}\right\}_{i=1, \ldots, j_{k}}$ has been selected. Take the Calderón-Zygmund decomposition of $f$ with respect to $2^{\ell+2 k}$, yielding intervals $\left\{Q_{k+1, i}^{\prime}\right\}_{i \in \mathbf{Z}_{+}}$. Let $\left\{Q_{k+1, i}\right\}_{i=1, \ldots, j_{k+1}} \subset\left\{Q_{k+1, i}^{\prime}\right\}_{i \in \mathbf{Z}_{+}}$be such that $\bigcup_{j} Q_{k+1, j} \subset \bigcup_{j} Q_{k, j}$ and $\left|\bigcup_{j} Q_{k+1, j}^{\prime} \backslash \bigcup_{j} Q_{k+1, j}\right|<\epsilon / 2^{k+1}$.

In this manner, we obtain intervals

$$
Q_{1,1}, \ldots, Q_{1, j_{1}}, Q_{2,1}, \ldots, Q_{2, j_{2}}, \ldots, Q_{m, 1}, \ldots, Q_{m, j_{m}},
$$

where $m$ is the largest integer such that $2^{\ell+2(m-1)}<\|f\|_{\infty}$. Let $\mathcal{N}$ be the union of all the $Q_{i, j}^{\prime}$ that are not on the list $(*)$. Note $|\mathcal{N}|<\epsilon$. 
Let $p \in[0,1] \backslash \mathcal{N}$. Let $Q_{p}$ be the smallest interval on the list $(*)$ that contains $p$. Note that

$$
M_{\mathrm{HL}}^{\Delta} f(p) \sim \frac{1}{\left|Q_{p}\right|} \int_{Q_{p}} f \sim \sum_{i, j} \frac{1}{\left|Q_{i, j}\right|}\left(\int_{Q_{i, j}} f\right) \chi_{Q_{i, j}}(p) .
$$

Also, for almost every $p \in[0,1] \backslash \mathcal{N}$, if $f(p)>4^{j} \geq 1$ then $p \in \cup_{i} Q_{j, i}$. Hence $p$ lies in at least $j$ intervals listed in $(*)$.

Let $R_{1}=Q_{m, 1}, R_{2}=Q_{m, 2}, \ldots, R_{j_{m}}=Q_{m, j_{m}}, R_{j_{m}+1}=Q_{m-1,1}, \ldots$, $R_{j_{m}+j_{m-1}}=Q_{m-1, j_{m-1}}, \ldots, R_{j_{1}+\cdots+j_{m}}=Q_{1, j_{1}}$.

If $j<k$, we have $\left|R_{k} \cap \bigcup_{j=1}^{k-1} R_{j}\right| \leq \frac{1}{2}\left|R_{k}\right|$ by the Calderón-Zygmund construction of the $Q_{i, j}$. To see this consider, say, $Q_{1,1}=[0,1]$. Now

$$
\frac{1}{\left|Q_{1,1}\right|} \int_{Q_{1,1}} f \leq 2^{\ell+1}
$$

Suppose $\left|Q_{1,1} \cap \bigcup_{i} Q_{2, i}\right|>\frac{1}{2}\left|Q_{1,1}\right|$. Then

$$
\begin{aligned}
\frac{1}{\left|Q_{1,1}\right|} \int_{Q_{1,1}} f & \geq \frac{1}{\left|Q_{1,1}\right|} \sum_{\substack{i: \\
\left|Q_{2, i} \cap Q_{1,1}\right| \neq 0}} \int_{Q_{2, i}} f \\
& >\frac{1}{\left|Q_{1,1}\right|} \sum_{\substack{i: \\
\left|Q_{2, i} \cap Q_{1,1}\right| \neq 0}} \int_{Q_{2, i}} 2^{\ell+2} \\
& =\frac{1}{\left|Q_{1,1}\right|} 2^{\ell+2}\left|Q_{1,1} \cap \bigcup_{i} Q_{2, i}\right|>2^{\ell+2} \cdot \frac{1}{2}=2^{\ell+1},
\end{aligned}
$$

contradicting $\frac{1}{\left|Q_{1,1}\right|} \int_{Q_{1,1}} f \leq 2^{\ell+1}$. Likewise, if $Q_{j, k}$ is on the list (*),

$\mid Q_{j, k} \cap$ union of the $Q_{r, s}$ following $Q_{j, k}$ on the list $(*)\left|\leq \frac{1}{2}\right| Q_{j, k} \mid$.

Hence $\left\{R_{i}\right\} \in \mathrm{CFC}\left(M_{\mathrm{HL}}^{\Delta}\right)$.

So there exists $\left\{R_{i}\right\} \in \mathrm{CFC}\left(M_{\mathrm{HL}}^{\Delta}\right)$ such that

$$
M_{\mathrm{HL}}^{\Delta} f(p) \sim \sum_{i} \frac{1}{\left|R_{i}\right|}\left(\int_{R_{i}} f\right) \chi_{R_{i}}(p)
$$

except on a set of measure less than $\epsilon$. Condition (i) is thus satisfied by $\left\{R_{i}\right\}$. We now show that condition (ii) is satisfied by the same Córdoba-Fefferman collection $\left\{R_{i}\right\}$ as well.

We have already demonstrated that, if $p \in[0,1] \backslash \mathcal{N}$ and $4^{j+1} \geq f(p) \geq$ $4^{j}>1$, then $p$ lies in at least $j$ of the $R_{i}$. Because in this case

$$
f(p)(\log (3+f(p)))^{2} \sim f(p)\left(\log \left(3+4^{j}\right)\right)^{2} \sim f(p) j^{2},
$$


we obtain

$$
f(p)(\log (3+f(p)))^{2} \lesssim f(p)\left(\sum_{i} \chi_{R_{i}}(p)\right)^{2} .
$$

If $f(p)<4$, then

$$
f(p)(\log (3+f(p)))^{2} \lesssim f(p) \lesssim f(p)\left(\sum_{i} \chi_{R_{i}}(p)\right)^{2},
$$

since $[0,1]$ is one of the $R_{i}$.

Let $\epsilon$ be small enough that

$$
\int_{0}^{1} f(\log (3+f))^{2} \sim \int_{[0,1] \backslash \mathcal{N}} f(\log (3+f))^{2} .
$$

Since

$$
\int_{[0,1] \backslash \mathcal{N}} f(\log (3+f))^{2} \lesssim \int_{[0,1] \backslash \mathcal{N}} f\left(\sum_{i} \chi_{R_{i}}\right)^{2} \lesssim\|f\|_{L(\log L)^{2}([0,1])}
$$

(the latter inequality holding by duality), $\|f\|_{L(\log L)^{2}([0,1])}=1$, and

$$
\int_{0}^{1} M_{\mathrm{HL}}^{\Delta} M_{\mathrm{HL}}^{\Delta} f \sim\|f\|_{L(\log L)^{2}([0,1])},
$$

we see that $\int_{0}^{1} M_{\mathrm{HL}}^{\Delta} M_{\mathrm{HL}}^{\Delta} f \sim \int_{0}^{1} f\left(\sum_{i} \chi_{R_{i}}\right)^{2}$, as desired.

Corollary 21. Let $\epsilon>0$ and $f \in L^{\infty}(Q)$. There exists $\left\{R_{i}\right\} \in \operatorname{CFC}\left(M^{\Delta}\right)$ such that:

(i) $M^{\Delta} f \sim \sum_{i} \frac{1}{\left|R_{i}\right|}\left(\int_{R_{i}}|f|\right) \chi_{R_{i}}$ on $Q$ except on a set of measure less
than $\epsilon$; and

(ii) $\int_{Q} M^{\Delta} M^{\Delta} f \sim \int_{Q}|f|\left(\sum_{i} \chi_{R_{i}}\right)^{2}$.

Proof. Apply Claim 20 and the Fubini theorem.

Lemma 22. Let $\left\{R_{i}\right\},\left\{S_{i}\right\}$ be in $\operatorname{CFC}\left(M^{\Delta}\right)$. Then

$$
\sum_{j} \frac{1}{\left|R_{j}\right|}\left(\int_{R_{j}} \sum_{i} \chi_{S_{i}}\right) \chi_{R_{j}} \lesssim\left(\sum_{i} \chi_{S_{i}}+1\right)\left(\sum_{i} \chi_{R_{i}}+1\right) .
$$

Proof. Let $j \in \mathbf{Z}_{+}$. It is enough to show that if $p \in R_{j}$, then

$$
\frac{1}{\left|R_{j}\right|} \int_{R_{j}} \sum_{i} \chi_{S_{i}} \lesssim\left(\sum_{i} \chi_{S_{i}}(p)+1\right) .
$$

Let $\left\{S_{i, \text { int }}\right\}$ denote the set of the $S_{i}$ contained in $R_{j}$. Let $\left\{S_{i, \text { ext }}\right\}$ denote the set of the $S_{i}$ strictly containing $R_{j}$. Note that, for some positive finite constant $C$,

$$
\frac{1}{\left|R_{j}\right|} \int_{R_{j}} \sum_{i} \chi_{S_{i, \mathrm{int}}} \leq C
$$


since $\int_{0}^{1}|\log x| d x=1$. Now, $\frac{1}{\left|R_{j}\right|} \int_{R_{j}} \sum \chi_{S_{i, \mathrm{ext}}}$ equals the number of $S_{i, \mathrm{ext}}$ strictly containing $R_{j}$, which equals $\sum \chi_{S_{i, \text { ext }}}(p)$. So

$$
\frac{1}{\left|R_{j}\right|} \int_{R_{j}} \sum_{i} \chi_{S_{i, \mathrm{ext}}} \lesssim \sum_{i} \chi_{S_{i, \mathrm{ext}}}(p) \lesssim \sum_{i} \chi_{S_{i}}(p)
$$

Hence, as desired,

$$
\frac{1}{\left|R_{j}\right|} \int_{R_{j}} \sum_{i} \chi_{S_{i}}=\frac{1}{\left|R_{j}\right|} \int_{R_{j}}\left(\sum_{i} \chi_{S_{i, \mathrm{int}}}+\sum_{i} \chi_{S_{i, \mathrm{ext}}}\right) \lesssim \sum_{i} \chi_{S_{i}}(p)+1 .
$$

Lemma 23. Suppose $f, g$, and $h$ are nonnegative measurable functions on $[0,1]$. Also suppose $\int_{0}^{1} f g^{2} \leq \int_{0}^{1} f g h$. Then $\int_{0}^{1} f g^{2} \leq \int_{0}^{1} f h^{2}$.

Proof. $\int_{0}^{1} f g^{2} \leq \int_{0}^{1} f g h$ implies

$$
\left\|f^{1 / 2} g\right\|_{2}^{2} \leq \int_{0}^{1} f g h \leq\left\|f^{1 / 2} g\right\|_{2}\left\|f^{1 / 2} h\right\|_{2}
$$

Hence

$$
\left\|f^{1 / 2} g\right\|_{2} \leq\left\|f^{1 / 2} h\right\|_{2}
$$

So $\int_{0}^{1} f g^{2} \leq \int_{0}^{1} f h^{2}$.

We now finish the proof of Lemma 19, and hence the proof of Lemma 18 as well. Without loss of generality, we assume $f$ is a nonnegative smooth function supported on $Q$.

Let $0<\epsilon=2^{-k}<1, k \in \mathbf{Z}_{+}$. Let $\mathcal{R}_{x, \epsilon}$ denote the set of dyadic rectangles in $Q$ of height $\epsilon$. Let the maximal operator $M_{x, \epsilon}^{\Delta}$ be given by

$$
M_{x, \epsilon}^{\Delta} f(p)=\sup _{p \in R \in \mathcal{R}_{x, \epsilon}} \frac{1}{|R|} \int_{R}|f|
$$

For sufficiently small $\epsilon, M_{x, \epsilon}^{\Delta} f \sim M_{x}^{\Delta} f$. Assume $\epsilon$ is indeed sufficiently small.

Now, since $\int_{Q} M_{x}^{\Delta} M_{x}^{\Delta} f \leq \int_{Q} M^{\Delta} M_{x}^{\Delta} f$ by hypothesis, we realize by Theorem 17 and Claim 20 that for some $\left\{R_{i}\right\} \in \operatorname{CFC}\left(M_{x, \epsilon}^{\Delta}\right)$ and some 
$\left\{A_{i}\right\} \in \operatorname{CFC}\left(M^{\Delta}\right)$,

$$
\begin{aligned}
\int_{Q} M_{x}^{\Delta} M_{x}^{\Delta} f & \sim \int_{Q} f\left(\sum_{i} \chi_{R_{i}}\right)^{2} \\
& \lesssim \int_{Q}\left(\sum_{i} \chi_{A_{i}}\right)\left(\sum_{j} \frac{1}{\left|R_{j}\right|}\left(\int_{R_{j}} f\right) \chi_{R_{j}}\right) \\
& \sim \int_{Q} f\left(\sum_{j} \frac{1}{\left|R_{j}\right|} \int_{R_{j}} \sum_{i} \chi_{A_{i}}\right) \chi_{R_{j}} \\
& \lesssim \int_{Q} f\left(\sum_{j} \chi_{R_{j}}\right)\left(\sum_{j} \chi_{A_{j}}\right),
\end{aligned}
$$

the latter inequality being justified by Lemma 22 and the fact that, letting $B_{j, k}=[0,1] \times\left[j \cdot 2^{-k},(j+1) 2^{-k}\right]$, we have

$$
\left\{A_{i} \cap B_{j, k}\right\}_{i \in Z_{+}, 0 \leq j \leq 2^{k}-1} \in \operatorname{CFC}\left(M_{x, \epsilon}^{\Delta}\right) .
$$

(Recall here that $\epsilon=2^{-k}$.)

By Lemma 23, we see that the above result

$$
\int_{Q} f\left(\sum_{i} \chi_{R_{i}}\right)^{2} \lesssim \int_{Q} f\left(\sum_{j} \chi_{R_{j}}\right)\left(\sum_{j} \chi_{A_{j}}\right)
$$

implies

$$
\int_{Q} f\left(\sum_{i} \chi_{R_{i}}\right)^{2} \lesssim \int_{Q} f\left(\sum_{i} \chi_{A_{i}}\right)^{2} \lesssim \int_{Q} M^{\Delta} M^{\Delta} f .
$$

Hence $\int_{Q} M_{x}^{\Delta} M_{x}^{\Delta} f \lesssim \int_{Q} M^{\Delta} M^{\Delta} f$, as desired.

Corollary 24. Suppose $f$ is a measurable function supported on $Q$. Then

$$
\int_{Q} M_{x} M_{x} f \lesssim \int_{Q} M M f+\int_{Q} M_{y} M_{x} f
$$

Proof. By (19) we have

$$
\int_{Q} M_{x}^{\Delta} M_{x}^{\Delta} \lesssim \int_{Q} M^{\Delta} M_{x}^{\Delta} f+\int_{Q} M_{y}^{\Delta} M_{x}^{\Delta} f .
$$

Applying Lemma 19, we see that

$$
\int_{Q} M_{x}^{\Delta} M_{x}^{\Delta} \lesssim \int_{Q} M^{\Delta} M^{\Delta} f+\int_{Q} M_{y}^{\Delta} M_{x}^{\Delta} f .
$$

Hence, by (17) we have

$$
\int_{Q} M_{x} M_{x} f \lesssim \int_{Q} M M f+\int_{Q} M_{y} M_{x} f .
$$


We are now in position to complete the proof of Theorem 14 by showing that

$$
\int_{Q}\left(M M \widetilde{f}+M_{y} M_{x} \widetilde{f}\right) \lesssim \int_{Q}\left(M M f+M_{y} M_{x} f\right) .
$$

We have $\int_{Q} M_{y} M_{x} \tilde{f} \lesssim \int_{Q} M_{y} M_{x} f$ by Corollary 10, and

$$
\int_{Q} M M \widetilde{f}=\int_{Q} M_{x} M_{x} \tilde{f} \sim \int_{Q} M_{x} M_{x} f \lesssim \int_{Q}\left(M M f+M_{y} M_{x} f\right)
$$

by Corollary 24. So

$$
\int_{Q}\left(M M \widetilde{f}+M_{y} M_{x} \widetilde{f}\right) \lesssim \int_{Q}\left(M M f+M_{y} M_{x} f\right),
$$

as desired.

\section{References}

[1] A. Córdoba and R. Fefferman, A geometric proof of the strong maximal theorem, Ann. of Math., 102 (1975), 95-100, MR 0379785 (52 \#690), Zbl 0324.28004.

[2] M.E. Gomez, A counterexample for the strong maximal operator, Studia Math., 78 (1984), 199-212, MR 0766716 (86k:42033), Zbl 0488.42021.

[3] P.A. Hagelstein, Córdoba-Fefferman collections in harmonic analysis, Pacific J. Math., 216 (2004), 95-109.

[4] P.A. Hagelstein, Local integrability of strong and iterated maximal functions, Studia Math., 147 (2001), 37-50, MR 1853476 (2002f:42018), Zbl 0983.42012.

[5] B. Jessen, J. Marcinkiewicz and A. Zygmund, Note on the differentiability of multiple integrals, Fund. Math., 25 (1935), 217-234, Zbl 0012.05901.

[6] E.M. Stein, Note on the class L log L, Studia Math., 132 (1969), 305-310, MR 0247534 (40 \#799), Zbl 0182.47803.

[7] E.M. Stein, Singular Integrals and Differentiability Properties of Functions, Princeton University Press, 1970, MR 0290095 (44 \#7280), Zbl 0207.13501.

[8] A. Zygmund, Trigonometric Series, Cambridge University Press, 1959, MR 0107776 (21 \#6498), Zbl 0085.05601.

Received June 9, 2000.

Department of Mathematics

BAYLOR UNIVERSITY

Waco, TEXas 76798

E-mail address: Paul_Hagelstein@baylor.edu 